\title{
FUTEBOL - Orchestrated Optical/Wireless Experiments Across Brazil and Europe
}

\author{
Daniel F. Macedo ${ }^{1}$, Luis H. Cantelli Reis ${ }^{1}$, José Marcos S. Nogueira ${ }^{1}$, \\ Cristiano B. Both ${ }^{2}$, Luiz da Silva ${ }^{3}$, Juliano Wickboldt ${ }^{4}$, Johann Marquez-Barja ${ }^{5}$ \\ ${ }^{1}$ Universidade Federal de Minas Gerais - Brazil \\ ${ }^{2}$ Unisinos - Brazil \\ ${ }^{3}$ Universidade Federal do Rio Grande do Sul - Brazil \\ ${ }^{4}$ Trinity College Dublin - Ireland \\ ${ }^{5}$ University of Antwerpen - Belgium \\ \{damacedo, luiscantelli,jmarcos\}@dcc.ufmg.br, cbboth@unisinos.br, \\ dasilval@tcd.ie, jwickboldt@inf.ufrgs.br, Johann.Marquez-Barja@uantwerpen.be
}

\begin{abstract}
This paper presents the experimentation facilities provided by the European Union-Brazil project "FUTEBOL - Federated Union of Telecommunications Research Facilities for an EU-Brazil Open Laboratory", from the point of view of the experimenter. The FUTEBOL testbeds to provide experimentation capabilities for optical-wireless experimentation, which is crucial for the development of $5 G$ technologies. The main offering of FUTEBOL is, thus, to be able to set up an experiment accross many network domains - be it optical, wireless, packet or cloud -, and to orchestrate them using tools such as COPA and ABNO. Further, FUTEBOL provides a set of services that can be instantiated with very little effort, in order to compose complex $5 G$ experiments in reduced time.
\end{abstract}

\section{Introduction}

Telecommunications networks remain largely segregated in the two domains of optical networks and wireless systems, and rarely do even researchers cross the boundary between the two. We argue that the evolutionary path [Raaf et al. 2014] and the needs of future telecommunication systems, be it for high data rate applications, for the Internet of Things (IoT) paradigm, or for aggressive backhaul requirements stemming out of cell densification, require the co-design of wireless access and optical backhaul and backbone. In this context, the EU-Brazil co-funded project FUTEBOL (Federated Union of Telecommunications Research Facilities for an EU-Brazil Open Laboratory) [FUTEBOL 2019] developed a converged control framework for experimentation on wireless and optical networks and to deploy this framework in federated research facilities, which will be made openly available to the whole research community.

The proliferation of small cells deployments increases frequency reuse and is one of the main means that allow for gains in mobile network capacity. On the optical network side, network function virtualization (NFV) and software-defined 
networks (SDN) are revolutionizing the way that network resources are managed. We view virtualization on the optical side and densification and capacity increase on the wireless access as major game changers in future networks that will deliver the best benefits when co-designed and experimented together. Therefore, this work describes a research infrastructure tailored to the needs of experimenters throughout the world, interested in issues that cross the boundary between wireless and optical networks. This infrastructure allows many types of experiments, as explained in more details in [Marques et al. 2017].

The focus of this paper is to present the FUTEBOL testbeds from the point of view of the user. In a nutshell, we present what are the capabilities of each testbed and what are the types of experiments that we envision in each of them. Then, we describe the FUTEBOL service catalogue and the orchestration tools, which are used by the experiment to compose and then control a multi-domain experiment. Finally, we describe a sample experiment using a graphic orchestrator called COPA.

The remainder of the paper is organized as follows. Section 2 presents each of the FUTEBOL testbeds as well as their offering. Section 3 describes the control framework, focusing on the aspects that are more relevant to the end-user. Section 4 shows a simple experiment. Section 5 concludes the paper.

\section{The FUTEBOL testbeds}

The FUTEBOL testbeds are heterogeneous testbeds, in which each institution provides a set of different resources. Because of that characteristic, FUTEBOL provides different types of equipment for experimentation in different $5 \mathrm{G}$ use cases. We describe below the main offerings of each testbed, as well as a glimpse of what type of experiments we envision to be run on each of them.

Iris TCD - the reconfigurable radio testbed at Trinity College Dublin (TCD) provides virtualized radio hardware, Cloud-RAN, Network Functions Virtualisation (NFV), and Software Defined Networking (SDN) technologies to support the experimental investigation of the interplay between legacy technologies, $5 \mathrm{G}$ radio and future networks. We offer the radio resources including 20 USRP N210s and 2 USRP X310s ceiling mounted nodes all equipped with SBX daughterboards, reaching frequencies between $40 \mathrm{MHz}$ and $4 \mathrm{GHz}$. These platforms are connected to a computational cloud, allowing us to deploy an array of computational environments, accessible through Fed4FIRE. To expose the functionality of these platforms for a variety of applications, we employ a variety of radio hypervisors that freely enable prototyping of wireless systems, as exemplified by GNURadio, srsLTE (an open-source 3GPP 4G stack from the Irish company Software Radio Systems), etc.

UNIVBRIS - The main focus at UNIVBRIS is to provide optical-packet orchestration, through a newer optical solution on an SDN-enabled network, and a virtualized infrastructure overlayed in an optical-packet path, which enables the experimental investigation using cross-layer algorithms that could be involved in 5G network research. Furthermore, experimenters can define physical parameters in an optical resource (BV-ROADM) and create an end-to-end (E2E) path using opticalpacket mapped by a tuple of ports and wavelengths. We deploy our infrastructure using OpenStack, ABNO and proprietary optical solutions from UNIVBRIS. 
UFES - The UFES testbed in light of the new generation of robotics as a service (e.g, robot localization and navigation, assistive robotics), has been built to support the requirements of real-time remote control of robots over a wireless-optical SDN-enabled network. It explores the field of cloud robotics control with the aid of computer vision that requires novel orchestration features offered by the FUTEBOL Control Framework (CF) such as i) flexible innovative vertical auto-scaling providing elasticity of Virtual Network Functions (VNF) using Openstack ii) orchestrating wireless, packet and optical networks to meet simultaneously low E2E latency and high bandwidth requirements, aiming to study and overcome the bottlenecks in the cloud-fiber-wireless domain, involving cyber-physical systems use cases with stringent requirements. Our target are production processes environments that need to test wireless control of industrial manufacturing, where URLLC communication services are expected, especially in the context of the Industry 4.0 (also known as Industrial IoT) vertical.

UFMG - UFMG's focus is on wireless experimentation, and how wireless technologies interact with cloud, packet and optical networks. As such, the UFMG testbed provides resources for experimentation of the so-called last mile technologies, that is, the technologies that connect the end-users to the core networks, using standards for both licensed $(4 \mathrm{G}$ ) and unlicensed (WiFi, ZigBee, Bluetooth) bands. The resources of the testbed allow the experimenters to program the network on layers 1 and 2 of the wireless protocol stack using USRP, while the implemented SDN protocols and NFV functionality provide programmability at layers 2 and above. Further, the testbed provides resources for IoT experimentation in an indoor environment.

UFRGS - The testbed is focused on devices for wireless research, Internet of things, sensor networks and optical-wireless convergence. With wireless experimentation (WiFi, 3G, 4G, 5G) including new protocols and technologies over programmable radio platforms (i.e., SDR), the testbed also provides optical-wireless convergence experimentation with its radio-over-fiber arrangement, where it's possible to create networks (up to the layer 2) using USRP through optical infrastructure.

VTT - The VTT testbed provides means for research and development by experimentation of wireless communication. The testbed may be enhanced by adding new hardware, for example targeted at $5 \mathrm{G}$ research. The testbed focus area is mainly shared spectrum access, LSA (Licensed Shared Access) being the current implemented and tested software, and SAS (Spectrum Access System) as a possible future improvement.

\section{The FUTEBOL Control Framework}

The deployment and control of experimentation slices, as well as the control of the experiment itself, is performed using tools that are available in the FUTEBOL control framework. The FUTEBOL control framework is divided into layers, as shown in Figure 1. The lowest layers are the common layers found in any testbed, namely the infrastructure layer and the virtualization layer. The infrastructure layer is composed of the physical links, compute resources as well as network equipment that is available for the experimenters. The virtualization layer provides the means to slice the physical infrastructure, supporting arbitrary topologies and multiple experiments. 


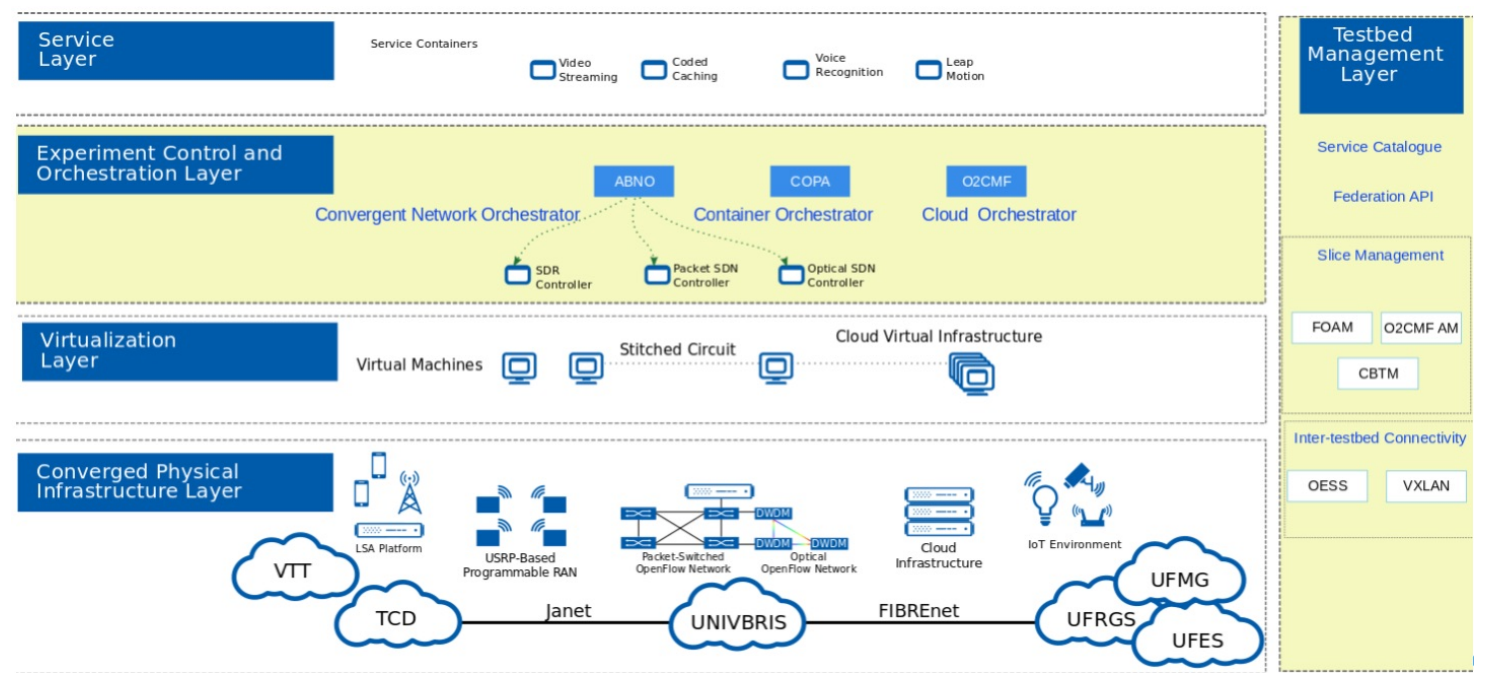

Figure 1. Structure of the FUTEBOL control framework

In this section we will describe two layers of the FUTEBOL CF, namely the testbed management layer and the orchestration layer.

\subsection{Testbed management layer}

The testbed management layer controls the allocation of the testbed resources. Further, it also offers a catalogue of services that provides virtual network functions (VNFs), services, and tools that facilitate converged experimentation. Each testbed will provide a different set of services in their catalogue, according to their key offering. Offering pre-installed services is very important for experimentation, since more sophisticated experiments may demand a long installation and setup time. For example, one may require to download files from github, compile them, and then configure the service.

The selection of the service that will be instantiated is based on the image that will be allocated on the virtual resource. For instance, a user may select a certain image that comes pre-loaded with an SDN controller, a video server, or even elements of the $5 \mathrm{G}$ core. In order to simplify the deployment of those serivces, each testbed provides a user guide that describes a basic experiment. The services come pre-loaded with configurations to realise those sample experiments.

\subsection{Orchestration layer}

The Experiment Control and Orchestration Layer allows the integrated manipulation of optical and wireless resources based on three tools. ABNO supports convergent network orchestration by interacting with the network controllers in the wireless and optical domains. COPA is an orchestration tool for containers developed to enable the live migration and monitoring of VNF across remote testbeds. O2CMF provides Cloud and NFV orchestration capabilities, such as automatic scaling of VNF based on pre-defined policies and monitoring of resource usage. Each tool is briefly described in the following paragraphs.

The ABNO architecture [Hammad et al. 2017] is based on IETF RFC7941 and enables network automation and programmability. The main motivation for 
developing $\mathrm{ABNO}$ as the network orchestrator in our $\mathrm{CF}$ is because most existing SDN controllers rely on monolithic software, offering insufficient flexibility for heterogeneous networks. This inflexibility has led to problems for network operators, such as vendor lock-in, lack of support for non-OpenFlow networks, and lack of support for end-to-end multi-domain networks.

$\mathrm{COPA}^{1}$ is a new orchestration and provisioning tool for containers, which we created to facilitate the migration and monitoring of virtual functions across testbeds. Live migration of full VM is only possible if they use a common hypervisor. However, we do not wish to force all testbeds to use the same hypervisor on their physical servers; moreover testbeds are already using different hypervisors for legacy reasons. Using containers to implement the virtual functions reduces the additional virtualization overhead for this second layer of abstraction.

O2CMF [Ceravolo et al. 2018] is based on OpenStack and is responsible for creating and managing VNF in a user's slice. To that end, it is composed by two elements: an AM and an orchestrator. The latter is offered to the user as a VM through of an orchestrator image. This orchestrator supports specifying an NFV experiment with monitoring and scaling policies using the TOSCA language,to send commands and to access any other VNF in the experiment.

Network functions and applications can be developed by using the Service Layer implemented in the FUTEBOL-CF architecture. This layer is responsible for deploying, for instance, SDR controllers (e.g., Open Air Interface Evolved Packet Core nodes, SRS LTE eNodeB implementation). Moreover, this layer is composed of the user's applications, e.g., a video server that will stream video during the experiment. Furthermore, this layer can provide experimentation tools to generate synthetic/realistic traffic and monitor the experiment network activity.

\section{An orchestrated inter-testbed experiment}

This experiment presents a scenario envisioned in Mobile Edge Computing [Hu et al. 2015] experiments. The experimental scenario is composed of two tiers of processing. The first and nearer to the user is the Fog, which has the characteristic of low processing power, but high network performance such as low network latency, low packet loss, and high network throughput. The second is the Cloud which has high processing power and typically worse network performance than the Fog. The main idea of this scenario is to monitor the quality of the network and computational resources, and compare the deployment and migration behavior of remote applications in such situations. To perform the monitoring and orchestration of the remote applications, we utilised COPA, a control framework application provided by FUTEBOL.

The experiment setup is illustrated in Figure 2. Currently, this experiment can be fully deployed in the FUTEBOL's testbeds. To access the hardware and virtual resources available by the consortium, we utilise JFed which provides a full list of the testbed resources and communicates directly with heterogeneous testbed control framework applications. JFed also enables the experimenter to reserve for a period of time a group of resources such as Raspberry Pis and Virtual Machines. During the reservation, we can also allocate Control Framework Services such as

\footnotetext{
${ }^{1}$ http://www.ict-futebol.org.br/wp-content/uploads/Deliverable5.1.pdf
} 


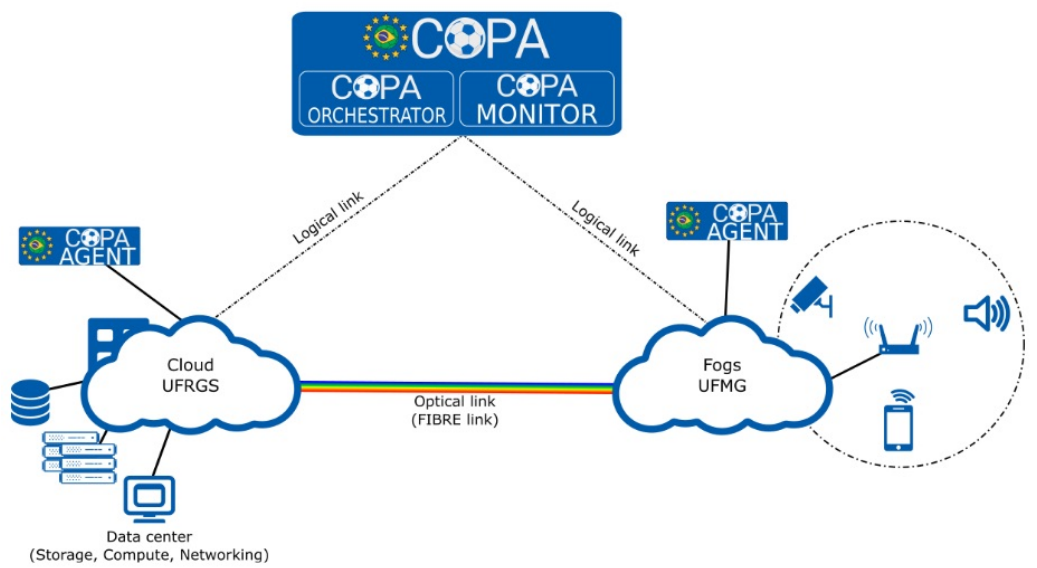

Figure 2. Experimental setup

COPA. Finally, inter-testbed connectivity is provided within certain testbeds (at the writing of this paper the links UFMG-UFRGS, UFRGS-Bristol, Bristol-TCD are operational).

Since this experiment is more focused on the use of the testbeds and not actually new scientific results, we show here a simple migration experiment. The user employs the COPA graphic user interface to perform a live migration of a container within testbeds. Each testbed will run a pool of COPA resources. COPA monitors the resource usage (link and $\mathrm{CPU}$ ) of the pools during the entire process. Such an experiment could be built in order to do live migration of IoT services, enabling the application to process the inputs within the required QoS levels. Our previous paper shows some application-related results for such an IoT scenario [Silva et al. 2017].

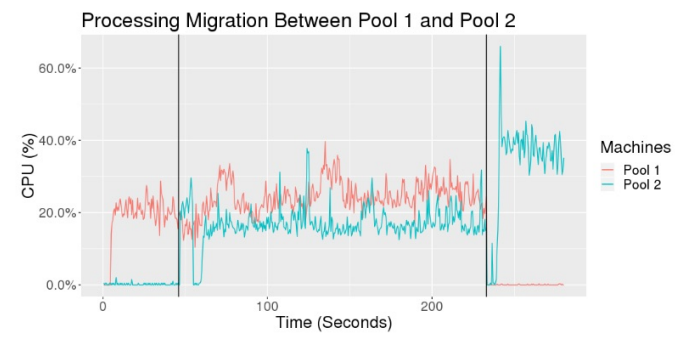

(a) CPU usage in the COPA pools.

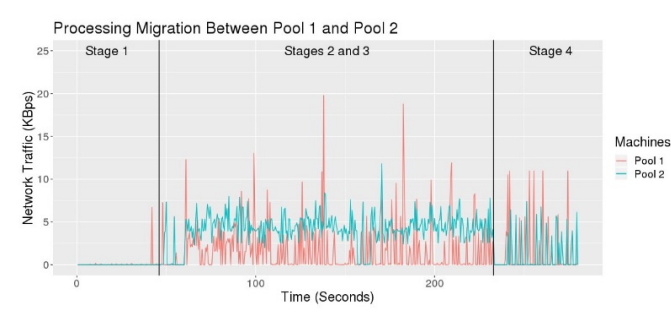

(b) Network traffic during the experiment.

Figure 3(a) shows the CPU load in Pool 1 and Pool 2 as the migration occurs. In the first part of the graph (first stage, defined by the period before the black vertical line), the usage of Pool $1 \mathrm{CPU}$ is around 20\%, while in Pool 2 is close to $0 \%$. When the migration process starts, the CPU use of Pool 2 increases because it is receiving the Object Detection container, but it is not running it yet. At this stage, object detection still takes place in Pool 1. Only in the final of the second stage the object detection (after the second vertical black line) occurs in Pool 2. In the final stage, the migration process is finalised and the CPU load of Pool 2 is around $40 \%$, while the load in Pool 1 is close to $0 \%$ as expected.

Figure 3(b) shows the network traffic of Pool 1 and Pool 2. In the left part of the figure (before the dark horizontal line), the traffic in Pool 2 is close to 0 bps. This 
happens because all the network load is in Pool 1, since it is running the Object Detection container. When the migration starts, Pool 2 must receive the Object Detection container, so the network traffic increases. This is shown in the graph in the curves between the two dark horizontal lines. Pool 1 has the same behavior, once it must send the container to Pool 2 for migration. When the migration ends, the network traffic of Pool 1 decreases, while the traffic of Pool 2 increases.

\section{Conclusions}

This paper presented the functionalities of the FUTEBOL testbeds and its framework from the point of view of the user. 5G research requires the orchestration of complex networks scenarios, spanning multiple network domains (such as optical, wireless, packet, and cloud) and over a wide area. The FUTEBOL project aims to support such experiments by providing heterogeneous physical resources among its testbeds. Those resources support complex services, via service catalogues. Further, the resources of multiple testbeds can be interconnected, thanks to the connectivity provided by RNP and Janet. Once the experiment is running, the experimenter will need to orchestrate those multiple domains so that the service's requirements can be accomplished. For that end, FUTEBOL supports two orchestrators, called COPA and ABNO.

\section{Acknowledgements}

The research leading to these results received funding from the European Commission H2020 programme under grant agreement no. 688941 (FUTEBOL), as well from the Brazilian Ministry of Science, Technology, Innovation, and Communication (MCTIC) through RNP and CTIC.

\section{References}

Ceravolo, I. et al. (2018). O2CMF: Experiment-as-a-service for agile Fed4Fire deployment of programmable NFV. In Optical Fiber Communication Conference.

FUTEBOL (2019). FUTEBOL - Federated Union of Telecom- munications Research Facilities for an EU-Brazil Open Laboratory. http://www.ict-futebol.org. $\mathrm{br} /$.

Hammad, A. et al. (2017). Demonstration of NFV content delivery using SDNenabled virtual infrastructures. In Optical Fiber Communication Conference.

Hu, Y. C., Patel, M., Sabella, D., Sprecher, N., and Young, V. (2015). Mobile edge computing - A key technology towards 5G. ETSI white paper, 11(11):1-16.

Marques, P. et al. (2017). Experiments Overview of the EU-Brazil FUTEBOL Project. In European Conference on Networks and Communications.

Raaf, B., Faerber, M., Badic, B., and Frascolla, V. (2014). Key technology advancements driving mobile communications from generation to generation. Intel Technology Journal, 18(1).

Silva, A. P., Abreu, B. A., Silva, E. B., Carvalho, M., Nunes, M., Marotta, M., Hammad, A., Silva, C. F. M., Pinheiro, J. F. N., Both, C. B., Marquez-Barja, J. M., 
and DaSilva, L. A. (2017). Impact of fog and cloud computing on an iot service running over an optical/wireless network testbed. In 2017 IEEE Conference on Computer Communications Workshops (INFOCOM WKSHPS), pages 535-540. 